Title:

VIDEO IMAGE PROCESSING FOR NUCLEAR SAFEGUARDS

Author(s):

C. A. Rodriguez

J. A. Howell

H. O. Menlove

C. M. Brislawn

J. N. Bradley

P. Chare

J. Gorten

Submitted to:

The Carnahan Conference on Security Technology Surrey, England

October 11, 1995

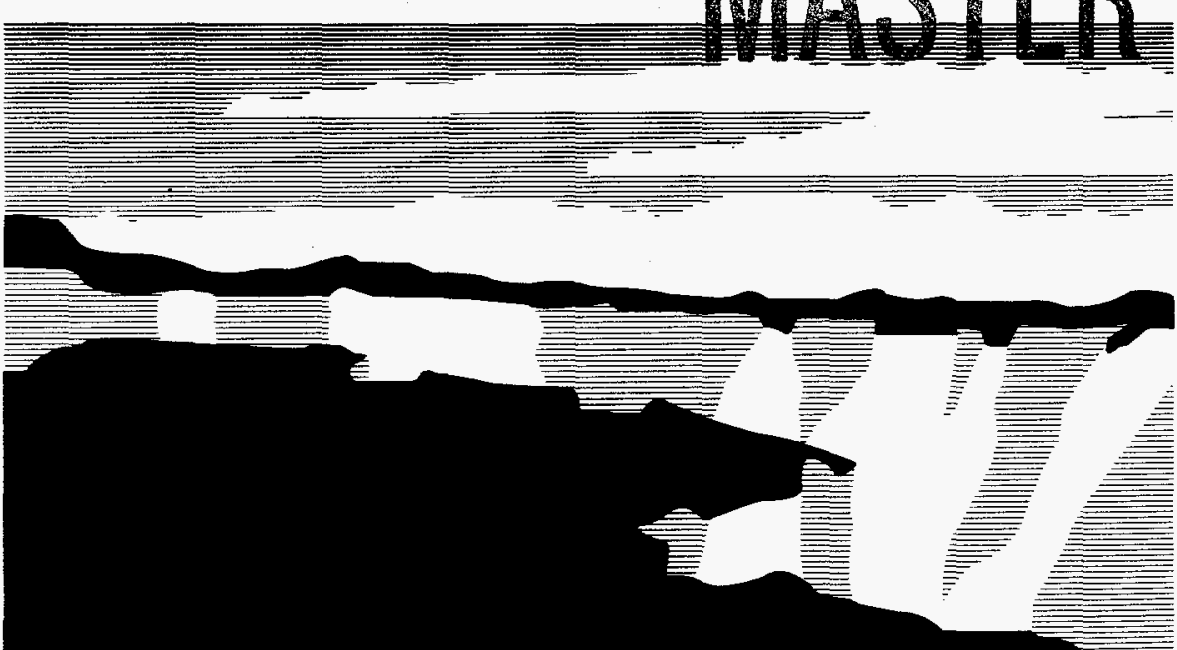

Los Alamos National Laboratory, an affirmative action/equal opportunity emplóyer, is operated by the University of California for the U.S. Department of Energy under contract W-7405-ENG-36. By acceptance of this article, the publisher recognizes that the U.S. Government retains a nonexclusive, royalty-free license to publish or reproduce the published torm of this contribution, or to allow others to do so, for U.S. Government purposes. The Los Alamos National Laboratory requests that the publisher identify this article as work performed under the auspices of the U.S. Department of Energy. 
DISCLAIMER

This report was prepared as an account of work sponsored by an agency of the United States Government. Neither the United States Government nor any agency thereof, nor any of their employees, makes any warranty, express or implied, or assumes any legal liability or responsibility for the accuracy, completeness, or usefulness of any information, apparatus, product, or process disclosed, or represents that its use would not infringe privately owned rights. Reference herein to any specific commercial product, process, or service by trade name, trademark, manufacturer, or otherwise does not necessarily constitute or imply its endorsement, recommendation, or favoring by the United States Government or any agency thereof. The views and opinions of authors expressed herein do not necessarily state or reflect those of the United States Government or any agency thereof. 


\section{DISCLAIMER}

Portions of this document may be illegible in electronic image products. Images are produced from the best available original document. 


\title{
Video Image Processing for Nuclear Safeguards
}

\author{
C. A. Rodriguez, J. A. Howell (NIS-7) \\ H. O. Menlove (NIS-5) \\ C. M. Brislawn, J. N. Bradley (CIC-3) \\ Los Alamos National Laboratory \\ P.O. Box 1663, Los Alamos, New Mexico 87545 USA \\ P. Chare, J. Gorten \\ The Commission of European Communities \\ EURATOM Safeguards Directorate \\ L-2985 Luxembourg
}

Abstract -- The field of nuclear safeguards has received increasing amounts of public attention since the events of the Iraq-UN conflict over Kuwait, the dismantlement of the former Soviet Union, and more recently, the North Korean resistance to nuclear facility inspections by the International Atomic Energy Agency (IAEA). The role of nuclear safeguards in these and other events relating to the world's nuclear material inventory is to assure safekeeping of these materials and to verify the inventory and use of nuclear materials as reported by states that have signed the nuclear Nonproliferation Treaty throughout the world. Nuclear safeguards are measures prescribed by domestic and international regulatory bodies such as DOE, NRC, IAEA, and EURATOM and implemented by the nuclear facility or the regulatory body. These measures include destructive and non destructive analysis of product materials/process by-products for materials control and accountancy purposes, physical protection for domestic safeguards, and containment and surveillance for international safeguards.

An example of materials analysis may be the destructive analysis of a sample to verify reported enrichment levels, or the nondestructive gamma-ray measurement of materials in storage for quantitative verification. Materials control and accountancy entails tracking materials through processing, fuel cycles, and reprocessing by implementing procedures and maintaining databases through the material's life cycle. These procedures may include the establishment of "boundaries" within a facility that serve as accountancy points. Inventory balances are then maintained in the databases and can be analyzed at discrete intervals for inventory differences that may indicate theft or diversion of nuclear materials.

Physical protection measures are used in domestic safeguards and include deterrents that protect materials from theft or tampering. The may include guards, -video, fences, and concrete barriers for facility protection. Containment and surveillance is used widely in international safeguards and may include paper, metal, or fiber optic seals for item monitoring; radiation detection devices; live video monitoring of facility areas; and recording of facility activities for later review. Recently this recording has been done by intelligent systems capable of unattended monitoring and scene analysis with triggered video recording that provides personnel with specific information about safeguards-related events.

In this presentation we will introduce digital video image processing and analysis systems that are being developed at Los Alamos for application to nuclear safeguards. Of specific interest to this audience will be the Inventory Verification System (IVSystem), an automated materials monitoring system; the Video Time Radiation Analysis Program (VTRAP), an integrated safeguards system; VideoTech, a surveillance system designed in conjunction with EURATOM for use in international safeguards; and we will introduce detector-activated predictive wavelet transform image coding used to significantly reduce the image data storage requirements for all of these unattended, remote safeguards systems.

\section{INTRODUCTION}

Until recently the use of video in US domestic safeguards has been largely limited to live video collected at key points within a facility and displayed to video monitors located in a central monitoring station. Typically these displays are in banks of nine or more monitors and are mainly used to view areas where other sensors have triggered an alarm. In international safeguards, still frames and video have been widely used for over ten years to record time lapse information about facility operations.

Early international still-frame systems simply recorded a still frame on film at predetermined intervals with the aid of an external, mechanical trigger. The film was periodically collected, developed, and reviewed by inspectors from the regulatory body. Current surveillance systems take advantage of personal computer technologies to drive the cameras 
and store the time-lapse imagery on analog or digital tapes. These images are collected by the computer system at programmed intervals, or when triggered by an external sensor, and then recorded on tape that is periodically collected and reviewed by the inspeetor.

The Los Alamos Inventory Verification System (IVSystem), Video Time Radiation Analysis Program (VTRAP), and VideoTech are next generation digital surveillance systems that use state of the art technologies for digital acquisition of surveillance imagery, image analysis, related data analysis, data compression, storage, and intelligent data review.

\section{The Inventory Verification System}

The IVSystem is a video-based surveillance application designed to facilitate a reduction in frequency of physical inventories for domestic nuclear materials vaults and longterm stores[1]. Based on video change detection, the IVSystem software continuously monitors the area of surveillance for changes. When a change, or an "event" occurs, it is automatically recorded, compressed, and stored along with an image that shows any changes introduced to the area during the event. Using this data the level of assurance that nuclear materials have not been tampered with or diverted is maintained, and the necessity for physical inventories is reduced. The IVSystem software is compatible with most SPARC architecture computers equipped with 1-4 processors, up to 512 MB internal RAM, and SCSI data storage devices that generally store $1.05 \mathrm{~GB}$ per device.

The IVSystem is part of an overall defense-in-depth protection strategy that relies on multiple layers of security to provide complementary nuclear safeguards measures. This surveillance system is viewed as being effective against the "insider threat" since it is not dependent on traditional monitoring devices that rely on receiving a positive signal to trigger an alarm. Such mechanisms, e.g., motion detectors, are perceived to be more vulnerable to insiders with a knowledge of the security apparatus. By contrast, difference coding of video images provides a constant verification of either the presence or absence of changes in the surveillance area. Under new Department of Energy (DOE) guidance on the use of "Alternative Security Measures," the IVSystem qualifies to reduce the required frequency of physical inventories and is instrumental to the DOE policy of keeping the radiation exposure of personnel "As Low As Reasonably Achievable (ALARA)."

\section{Principle of Operation}

IVSystem video change detection is based on the analysis of differences between video frames. Several algorithms are used, depending on environment and operational requirements including pixel to pixel differences, linear prediction analysis, and wavelet transform analysis. The most simple method is the pixel to pixel analysis, where each pixel is compared with a corresponding pixel from a previous frame to determine the amount of change that has occurred. The overall change is then analyzed to determine if the change in the scene exceeds configuration parameters. This change detection method is the commonly used by the IVSystem because it is simple, extremely sensitive, fast, and can determine whether an item has been added to the area, removed from the area, or simply moved within the area. Figure 1 illustrates the results of pixel analysis when an access has been made to nuclear materials storage container. The upper left frame shows a vault area before access; the middle frame shows the same area after the access; and the lower right frame shows the container that has been accessed in black.

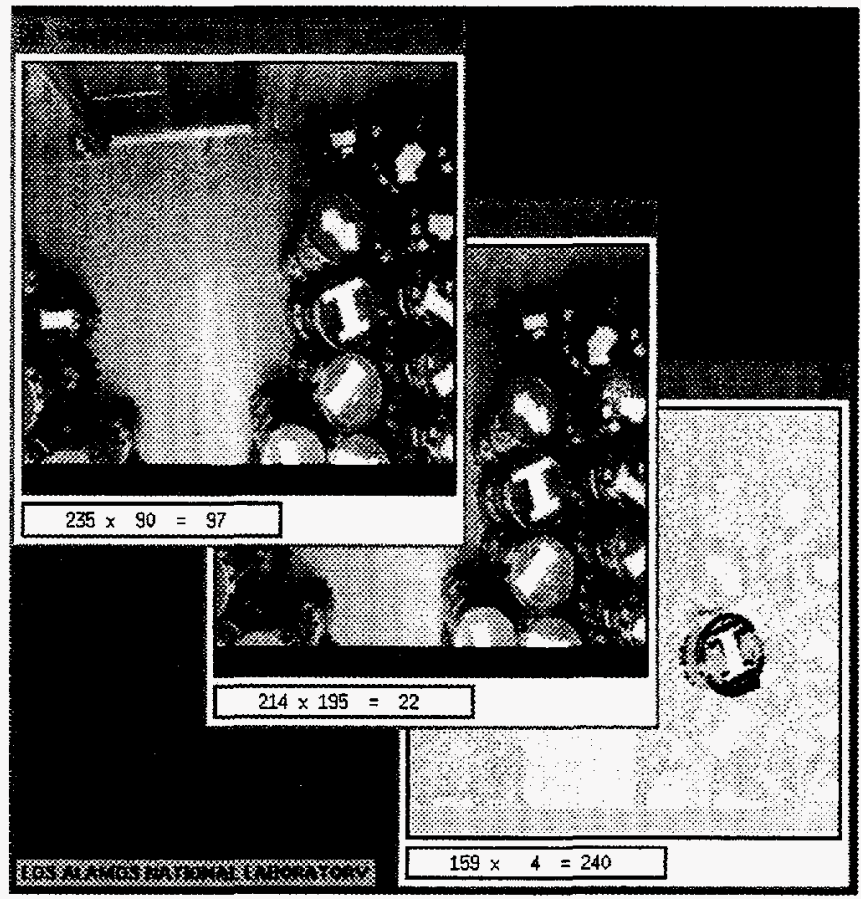

Figure 1: Pixel differences can detect changes in a scene that may be overlooked by the human eye.

Linear prediction analysis can be used when conditions such as changing natural light are present in the area of surveillance. Using this method changes in the overall scene, such as lighting changes, are discounted while local changes, such as the movement of an object, are detected. This method has an obvious advantage for some applications but does require more processing time per frame. Although this method has been developed for use with the IVSystem, no current field installations have required its use. 
Regardless of the change detection method used, the IVSystem can be configured to automatically record digital video of the event that has been detected. Wavelet transform analysis is used to detect and store differences between scenes in a highly compressed format. This video can then be transmitted or stored for later review. The advantage of the wavelet transform method over other common compression methods is that it produces very high quality in the reconstructed images while still providing high compression ratios.

\section{Field Installations}

The Inventory Verification System is currently installed at the Idaho National Engineering Laboratory (INEL), Rocky Flats Environmental Technology Site (RFETS), Savannah River Site (SRS), Argonne National Laboratory/West (ANL/ W), Arzamas-16 (a Russian defense facility), and at the Institute for Physics and Power Engineering (IPPE, a Russian nuclear research facility). The INEL installation has been our primary beta test site and has provided test data and user feedback since 1992. At INEL the IVSystem is configured with six cameras and monitors a Uranium storage vault and a field of annular storage wells used to store the same material.

RFETS, SRS, and ANL/W are new installations of the IVSystem and will monitor Plutonium storage vaults (or vault-type rooms) as well as interim storage areas. These systems will range in configuration from 1 to 13 cameras and are planned to be operational by early 1996.

At Arzamas-16 the IVSystem is being used as part of a demonstration of integrated Materials Protection, Control, and Accountability (MPC\&A). The IPPE installation is part of a similar integrated demonstration that will provide a model of enhanced MPC\&A for other power production and research facilities within Russia. While the IPPE installation is part of a demonstration of technologies, the facility is an operational research facility and the IVSystem will be used as a permanent materials monitoring system. The initial installation for the August \& September 1995 demonstrations will monitor one critical assembly area and associated Plutonium storage vault. Following the demonstrations the installation will be expanded to monitor an additional critical assembly area and two additional Plutonium storage vaults.

\section{Video Time Radiation Analysis Program}

Complete nuclear facility monitoring for nuclear safeguards requires the use of multiple sensors including cameras, radiations detectors, and other sensors all operating in unattended mode for time periods up to 3 months. The time interval for data collection must be small enough to avoid missing a critical event in the area being monitored. In some cases a 1-2 second time interval is necessary to provide the required temporal resolution. In others, a 5-6 minute interval may be adequate. As the number of sensors increases and the time interval between collected data points decreases, the amount of accumulated data becomes large enough to require computer based techniques to assimilate and evaluate the information. The Video Radiation Analysis Program (VTRAP) is designed to automate the collection and integration of video, radiation measurement, and other sensor data into large data bases for computer-based analysis with the objective of detecting anomalous activities within the facility[2].

To develop and evaluate the VTRAP concept, a test-bed was established in a controlled radiation laboratory at the Los Alamos National Laboratory. The equipment includes a nuclear materials vault, 2 neutron slab totals detectors, a neutron coincidence counter, and digital video camera(s). Figure 2 shows the test bed configuration.

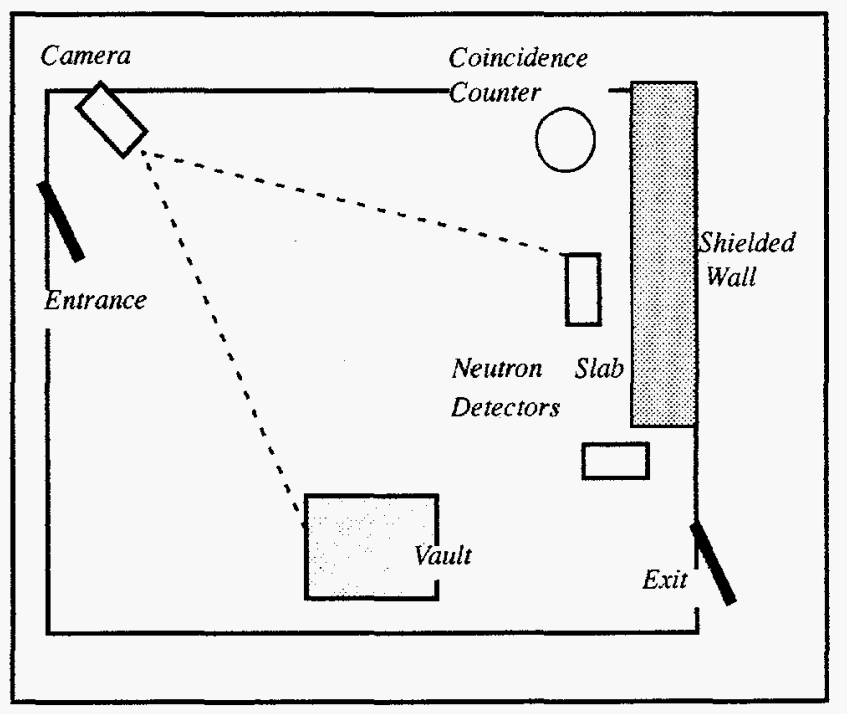

Figure 2: VTRAP Test-bed Configuration

The vault is considered a hardened and secure storage room or device that houses Californium- 252 source $(s)^{1}$ or other nuclear materials. Each neutron slab totals detector consists of six 1-by-12 inch ${ }^{3} \mathrm{He}$ (helium) tubes placed into a moderating slab of high-density polyethylene. The coincidence counter is a High-Level Neutron Coincidence Counter

1. A source is a small, quantified amount of radioactive material commonly used for instrument calibration or research in the area of nondestructive assay of nuclear materials. 
in a "well" configuration[3]. The digital video camera is a CCTV device with 512-by-512 resolution. Data are collected from each of these devices by control programs running on a SPARC architecture single processor computer.

The object of complete nuclear facility monitoring is to detect anomalous activity, that is, an activity that is inconsistent with reported facility operations or an activity that deviates from normal procedural operations. For the VTRAP test-bed four scenarios representing two normal activities and two anomalous activities have been defined.

Normal Scenario \#1: One or more personnel walking through the monitored area, entering through door marked entrance in Figure 2, and leaving through door marked exit.

Normal Scenario \#2: One or more personnel enter the monitored area, remove a source or other nuclear material from the storage vault, walk past the neutron slab totals detectors, and deposit the source in the coincidence (well) counter. After allowing the count to be completed, the source is returned to the storage vault.

Anomalous Scenario \#1: One or more personnel enter the monitored area, remove a source or other nuclear material from the storage vault and exit the room.

Anomalous Scenario \#2: One or more personnel enter the monitored area, remove a source or other nuclear material from the storage vault, step out of camera view, switch the material with an empty container, return the empty container to the vault, and exit the room.

During day-to-day operation of the test-bed facility we simulate nuclear facility operations by performing one or more of the above scenarios. During the simulation, sensor data is collected from each of the four sensors described above, correlated, and processed to determine if the event is within normal operations or if it is anomalous.

\section{Neural Network Data Analysis}

A neural network is an iterative numerical technique that facilitates the solution of a number of different types of problems including pattern matching and data categorizing. Neural networks are so named because of their similarity to actual biological neurons and their connections. The neural network is first "trained" to distinguish between normal and anomalous events using a control data set, and then is employed to detect anomalous activity using data collected by the VTRAP sensors. Several steps are performed to prepare the neural network to detect anomalies. Using the control data set we
- Identify each "event,"

- Extract features of the events,

- Use these features to generate a training set for the neural network, and

- Train the neural network to identify anomalies.

Once the neural network is trained, similar steps are taken to identify an anomalous event.

- Identify each "event,"

- Extract features of the events,

- Use these features to generate a "recall" data set, and

- Use the neural network to process the recall data set and identify anomalous events.

\section{Event Identification}

The first step to identifying anomalous activity is to identify individual events such as any of our above scenarios. Event identification may key off of video data if all activities in the monitored area are of interest, or it may key off of neutron data if only events with nuclear materials involved are of interest. Once identified, the event data are extracted from the complete data set.

\section{Feature Extraction}

Next a number of features that characterize these events are extracted from the event data set for use in the training, testing, and recall steps of the neural network analysis. Features are extracted using the following scheme. When an event is identified the time of the event is recorded and features such as count rates from neutron detectors and change levels from video data are calculated. These features are then incorporated into training, test, and recall data sets that are used by the neural network.

\section{Neural Network Training and Recall}

The neural network used by VTRAP to model operational scenarios and recognize patterns is a simple back propagation network with $n$ inputs and $m$ outputs. The $n$ inputs correspond to the $n$ features extracted, and the $m$ outputs correspond to the $m$ types of operational scenarios. During the training step control data sets are processed that contain distinct examples of the $m$ types of operational scenarios. These results are used to tune the neural network to produce results that fall within an acceptable probability of certainty. Recall data sets are those obtained in normal operational conditions and are processed by the neural net after the training and testing have produced acceptable results. 


\section{VideoTech}

VideoTech is a surveillance system based on advanced video technologies that is currently being developed by Los Alamos National Laboratory and the Euratom Safeguards Directorate for use in international safeguards applications. This system will be largely based on detection technologies developed for the Inventory Verification System, but will expand on these technologies in response to the more rigorous requirements for international safeguards surveillance systems. These additional requirements include region of interest processing, data authentication, data storage suitable for truly unattended operation, and tamper indicating system housings suitable for less than optimal operating environments.

\section{Region of Interest Processing}

Surveillance for international safeguards applications often entails wide area monitoring in addition to the storage area monitoring performed by the Inventory Verification System. An example is a fuel cask storage facility that may have shipping doors, a crane for movement of the fuel cask, fuel cask storage area, personnel access doors, staging areas, and possibly other areas used for facility operations. The object of surveillance in this example is the fuel cask and only movements related to the cask are of interest to the regulatory body. The region of interest software being developed by Los Alamos provides a flexible method for defining areas of surveillance within a scene and controlling what actions are to be performed by the unattended surveillance system upon detection of an event.

The VideoTech region of interest system allows the free hand drawing (using a mouse over a captured image of the surveillance area) of regions, or zones around areas of interest. Zones may be green, yellow, or red depending on the actions to be performed in case of event detection. A green zone would simply record a log entry indicating that access to this area has been detected. A yellow zone would be used for an area of intermediate interest. If accessed, a log entry would be made and one video frame would be stored representing the beginning of the event. A red zone would be used when a video recording of the event is required. Areas not designated by zone are not monitored by the system.

In our example fuel cask storage area personnel access doors may be designated as green zones so that all access to the area are noted. Staging or other work areas may be of no interest to the safeguards regulatory body and may be left unmonitored. The shipping doors through which a fuel cask must pass may be designated a yellow zone to document any usage of these doors, and the primary objects of interest, the crane and the fuel cask itself, would be designated as red zones. Any activity detected that exceeds configuration parameters would trigger the automatic recording, compression, and storage of a video representing the activity.

Another use for region of interest processing is where directional flow of materials, vehicles, or activities is of interest. An example would be a process area where raw materials entered by one channel and product was removed by another channel. Using directional logic multiple regions of interest can be evaluated to detect any directional movement that is inconsistent with normal facility operations. In this way normal facility operations are left unrecorded, and anomalous activities of interest to the safeguards regulatory body are detected and recorded.

\section{Data Authentication}

Important to the nuclear safeguards regulatory body is the authenticity of data collected by the unattended video surveillance system. When video data is transmitted or collected for review there must be some mechanism that ensures the data presented is exactly the same as the data collected. VideoTech will incorporate the Data Embedding authentication method developed at Los Alamos National Laboratories, a system that utilizes inherent noise, characteristic of all video images, to encode authenticating information into the image.

Data Embedding relies on the $5-10 \%$ inherent noise that exists in each digital video frame. Noise is defined as some deviation from the true pixel integer representation, often located in the lower bits of the byte(s) representing the integer in the computer. Using image analysis methods this noise can be located, and then replaced with real information, such as the date, location, and time of the frame, with out changing the quality of the video frames. This information is then secured with a key, similar to a password used to access a computer system. If the video data has been altered the compromise is detected through use of the key.

The Los Alamos Data Embedding method has been found to be highly compatible with the Wavelet Transform Image Coding compression method used by the Inventory Verification System, VTRAP, and VideoTech. Integrating the two methods we have achieved highly compressed authenticated data with excellent reconstructed imagery.

\section{Data Compression}

Common to all of these automated nuclear safeguards systems is the need to store large quantities of video and other sensor data, and with regard to video data, to achieve a minimal loss of quality in the reconstructed image[3]. To achieve the required compression ratios and maintain minimal loss of 
quality in the reconstructed images, we adopted a wavelet transform compression algorithm based on predictive image coding strategies currently being developed at Los Alamos.

A prediction of each frame is made based on the previous frame, and the error residual (the difference between the actual and predicted frames) is coded for storage or transmission or both. The change detection algorithm plays an important role in the compression process in that it is used to adjust the quality level in the frame-difference coder based on the activity in the scene. By using relatively low quality coding on frames with little activity, very large compression ratios can be realized.

\section{Discrete Wavelet Transform Image Coding}

Compression of image frames is based on discrete wavelet transform (DWT) sub-band coding and adaptive uniform scalar quantization. The sub-band coder is a two-channel perfect-reconstruction multi-rate filter bank (PR MFB) with linear-phase FIR filters corresponding to a family of regular, bi-orthogonal wavelets; this same PR MFB is used in the FBI's wavelet/scalar quantization (WSQ) standard for coding digital fingerprint images $[3,4]$. The filters are applied to row and column vectors of an image using symmetric extrapolation at the image boundaries; this "symmetric wavelet transform" technique is detailed in [5]. The resulting two-dimensional transform is then cascaded down five levels to produce the 16-band octave-scaled decomposition shown in Figure 3. Note that this particular decomposition has fewer sub-band splittings than the decomposition employed in Ref. [3], which was tailored to preserve very fine details in fingerprint images at the expense of slightly increased ringing distortion near edges.

Once the DWT decomposition of an image has been formed, a bank of 16 uniform scalar quantizers is designed for quantizing the wavelet coefficients in the 16 sub-bands, based on the following optimal bit-allocation problem: minimize the scalar quantization distortion model

$$
D(\mathbf{r})=\sum_{k=1}^{16} \frac{1}{m_{k}} \sigma_{k}{ }^{2} 2^{-2 r_{k}},
$$

subject to the user-imposed constraint on the overall bit rate

$$
\sum_{k=1}^{16} \frac{r_{k}}{m_{k}}=R \text {. }
$$

Here, $m_{k}$ is the down-sampling ratio for the $k^{\text {th }}$ sub-band; e.g., the bands between $\frac{\pi}{2}$ and $\pi$ in Figure 3 have been down-sampled 2:1 in both the horizontal and vertical directions, so $m_{k}=4$ for those bands. The other bands have down-sample factors that are higher powers of 4 . The Lagrange multiplier solution to this bit allocation problem is

$$
r_{k}=R+\frac{1}{2} \log _{2}\left[\frac{\sigma_{k}^{2}}{\prod\left(\sigma_{j}^{2}\right)^{1 / m_{j}}}\right]
$$

which involves a "scaled" geometric mean of the sub-band variances. The derivation of this result is based on the assumption that

$\sum_{k} \frac{1}{m_{k}}=1$

a relation that holds for any critically sampled sub-band decomposition. An iterative procedure is used to eliminate sub-bands with negative "optimal" bit rates and ensure nonnegativity of the $r_{k}$ 's.

The integer quantizer indices generated by the scalar quantizers are then compressed by zero-run-length and Huffman coding. Low bit-rates for the high-frequency sub-bands produce long runs of zeros in those portions of the sub-bands corresponding to smooth regions of the image; this is the principal source of additional lossless coding gain over and above the lossy gain produced by wavelet coefficient quantization. Note that this technique makes use of the good space/ frequency localization properties of sub-band image coding with short FIR filters.

\section{Predictive Coding of Image Sequences}

Now consider coding a sequence or "run" $x_{0}, x_{1}, x_{2}, \ldots$ of consecutive images taken by a still surveillance camera monitoring a scene of interest. The goal is to provide an uninterrupted record of surveillance images at very low storage costs during periods when the scene is inactive, yet obtain high-quality imagery during active periods. The initial frame rate is therefore assumed to be "low," e.g., one frame every few seconds. The initial frame in a run, $x_{0}$, is compressed using nonpredictive coding. Because no motion is presumed to occur in the scene in the inactive state, we use a simple first-order predictor and code the difference frames for subsequent time-steps, $x_{i}-\hat{x}_{i-1}$, where $\hat{x}_{i-1}$ is the recon- 
structed, quantized approximation of $x_{i-1}$. The DWT is a linear transformation, so the transform $d_{i}$ of the $i^{\text {th }}$ difference image can be computed by taking the difference of the DWT's, $a_{i}$ and $\hat{a}_{i-1}$, of $x_{i}$ and $\hat{x}_{i-1}$ :

$$
d_{i}=\mathrm{DWT}\left(x_{i}-\hat{x}_{i-1}\right)=a_{i}-\hat{a}_{i-1}
$$

This eliminates the need to perform an inverse DWT inside the prediction feedback loop, improving on the predictive scheme described in Ref. [6]. Note that $a_{i}$ consists of 16 separate spatial frequency sub-bands, as shown in Figure 3, so " $d_{i}$ " denotes the difference signals for the 16 DWT subbands. The transformed difference $d_{i}$ is quantized by the bank of uniform scalar quantizers described above and Huffman coded for transmission; the block diagrams for this predictive encoder/decoder system are shown in Figs. 4 and 5.

While the scene is inactive, the frame-to-frame differences are extremely small and can be coded at a very low bit rate with little perceptible distortion, allowing a continuous record to be archived at a low storage cost. Of course, as soon as an event occurs and the state becomes active, a much higher bit rate is needed to record the event accurately. A major advantage of adaptive scalar quantization is that the user-imposed overall bit rate $R_{i}$ can be changed on the fly. In most image-sequence coding scenarios, it is very difficult to maintain constant perceptual image quality by automatically measuring scene content and adaptively modifying the quantizer bit rates. In the specialized remote surveillance scenario under consideration, however, image coding conditions divide naturally into active/inactive states, and there is a straightforward criterion for deciding when and by how much quantizer bit rates should be changed.

In Figure 4 we have indicated how the transformed difference image, coupled with input from other sensors (e.g., motion or radiation sensors), can be fed to an event detector that automatically triggers a higher bit rate (and possibly an increased frame rate) in response to an event detection. Bit rates for the inactive state can be preset to minimize the amount of data required to verify that the scene remained unchanged when sensors detected no activity, and bit/frame rates for active states can be set high enough to provide necessary resolution for monitoring activities. This accomplishes our dual goals of providing uninterrupted coverage of the scene at very low storage cost during inactive states while still producing high-resolution coverage of active states. 


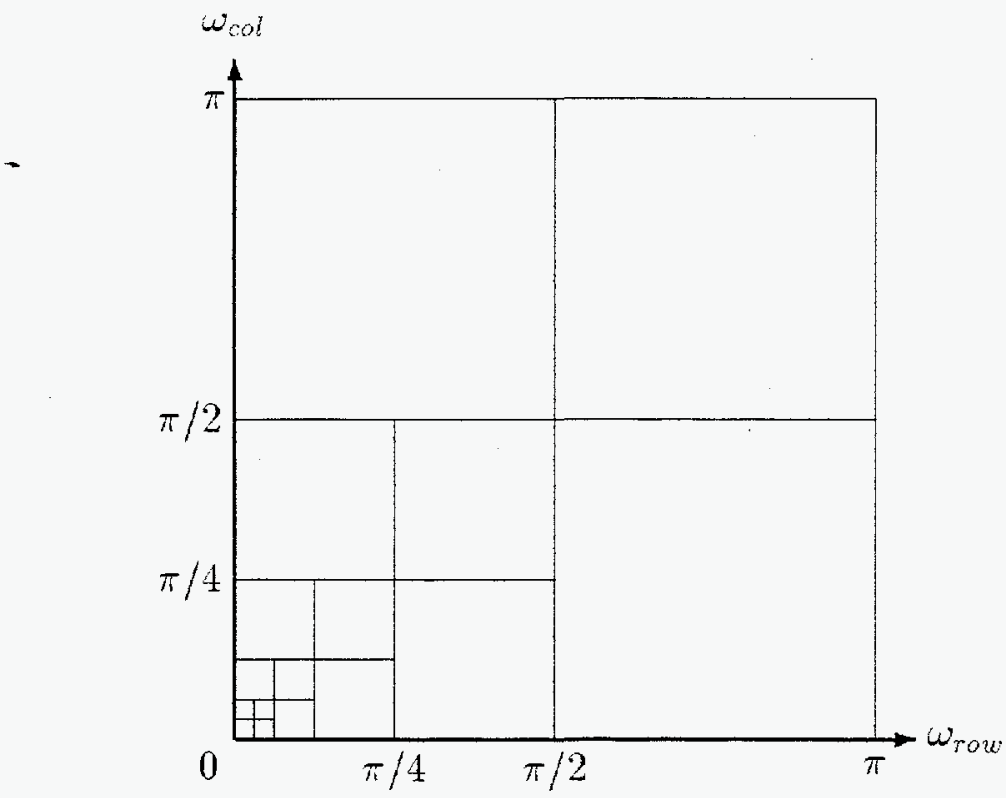

Figure 3: Approximate frequency passbands for five-octave DWT decomposition.

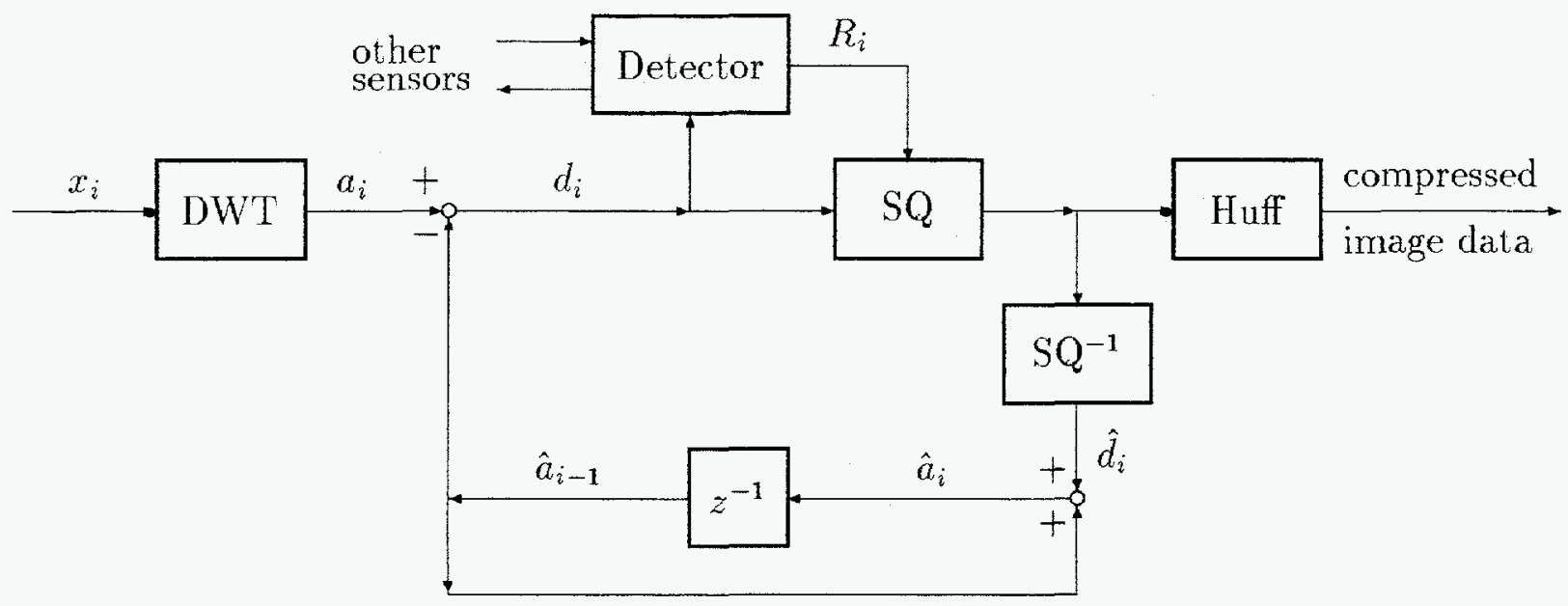

Figure 4: Detector-activated predictive wavelet transform image coder.

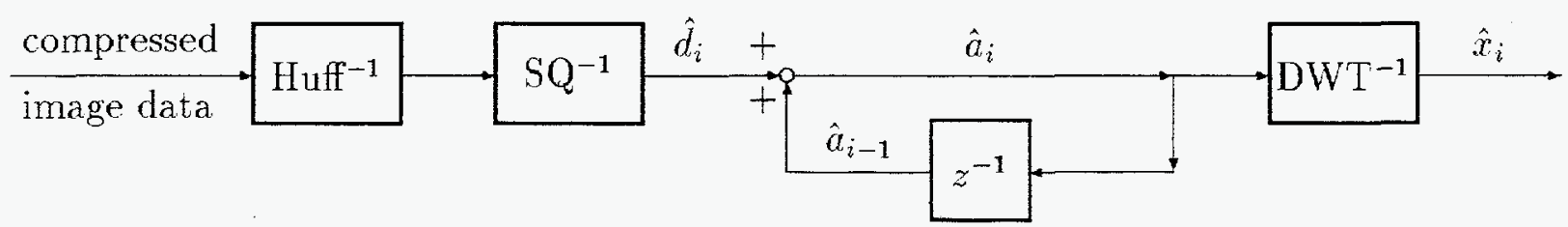

Figure 5: Predictive wavelet transform image decoder. 


\section{REFERENCES}

1. C. A. Rodriguez, Video Image Processing for Nuclear Safeguards: The Experimental Inventory Verification System, TechnicarReport N4/93-343, 1993.

2. J. A. Howell, C. A. Rodriguez, R. Whiteson, J. W. Eccelston, J. K. Halbig, H. L. Menlove, S. F. Klosterbuer, M. F. Mullen, Safeguards Applications of Pattern Recognition and Neural Networks, in Proceedings of the IAEA Symposium on International Safeguards, (Vienna, Austria), March 1994.

3. M. S. Krick and H. O. Menlove, The High-Level Neutron Coincidence Counter (HLNCC): User's Manual, Los Alamos Scientific Laboratory report LA-7779-M (1978).

4. J. N. Bradley, C. M. Brislawn, J. E. Brown, C. A. Rodriguez, L. A. Stoltz, Video Imaging for Nuclear Safeguards, in Proceedings of the IEEE Data Compression Conference, (Snowbird, Utah), April, 1994.

5. T. Hopper, C. Brislawn, and J. Bradley, WSQ GrayScale Fingerprint Image Compression Specification, IAFIS-IC-0110V2, Criminal Justice Information Services Division, Federal Bureau of Investigation, February 1993.

6. J. Bradley, C. Brislawn, and T. Hopper, The FBI Wavelet/Scalar Quantization Standard for Gray-Scale Fingerprint Image Compression, in Proceedings of the Conference for Visual Information Processing II, vol. 1961 of the Proceedings of the SPIE, (Orlando, FL), pp. 293-304, Society of Photo Optical Instrument Engineers, April 1993.

7. C. M. Brislawn, Classification of Nonexpansive Symmetric Wavelet Transforms, Technical Report LA-UR93-2236, Los Alamos National Laboratory, May 1993.

8. J. N. Bradley and C. M. Brislawn, Applications of Wavelet-Based Compression to Multidimensional Earth Science Data, in Proceedings of the Space Earth Science Data Compression Workshop, no.3191 in NASA Conference Publication, (Snowbird, UT), pp. 13-24, National Aeronautics and Space Administration, April 1993. 